\title{
A metabolic mechanism analysis of Fuzheng-Huayu formula for improving liver cirrhosis with traditional Chinese medicine syndromes
}

\author{
Ya-nan SONG ${ }^{1}$, Jian $\mathrm{CHEN}^{1}$, Fei-fei CAI ${ }^{1}$, Yi-yu LU ${ }^{1}$, Qi-long $\mathrm{CHEN}^{1}$, Yong-yu ZHANG ${ }^{2}$, Ping LIU $^{3, *}$, Shi-bing SU ${ }^{1, *}$ \\ ${ }^{1}$ Research Center for Traditional Chinese Medicine Complexity System, Shanghai University of Traditional Chinese Medicine, Shanghai \\ 201203, China; ${ }^{2}$ Research Center for Traditional Chinese Medicine and System Biology, Shanghai University of Traditional Chinese \\ Medicine, Shanghai 201203, China; ${ }^{3}$ E-institutes of Traditional Chinese Internal Medicine, Shanghai Municipal Education Commission, \\ Shanghai 201203, China
}

\begin{abstract}
Fuzheng-Huayu formula (FZHY), a Chinese herbal mixture prescription, has been proven effective in treating liver fibrosis and cirrhosis in both clinical trials and animal experiments. In this study we assessed the metabolic mechanisms of traditional Chinese medicine (TCM) syndrome-based FZHY treatment in liver cirrhosis (LC). A total of 113 participants, including 50 healthy controls and 63 LC patients, were recruited. According to the diagnosis and differentiation of the TCM syndromes, the LC patients were classified into 5 TCM syndrome groups including the liver stagnation syndrome (LSS), spleen deficiency and damp overabundance syndrome (SDDOS), damp-heat accumulation syndrome (DHAS), liver-kidney Yin deficiency syndrome (LKYDS), and blood stagnation syndrome (BSS), and administered FZHY for 6 months. FZHY treatment significantly decreased serum levels of hyaluronic acid (HA), a biochemical marker for LC, as well as TCM syndrome scores (the TCM syndrome scores were decreased in all the groups with significant decreases in the LSS and LKYDS groups). Furthermore, FZHY treatment gradually shifted the metabolic profiles of LC patients from a pathologic state to a healthy state, especially in LC patients with LSS and LKYDS. Twenty-two differently altered metabolites (DAMs) were identified, including carbohydrates, amino acids, fatty acids, etc with 9 DAMs in LSS patients, 9 in LKYDS patients, and 4 in other patients. The metabolic pathways involved in the conversion of amino acids and the body's detoxification process were regulated first, followed by the pathways involved in the body's energy supply process. In conclusion, the evaluation of the effect of TCM syndrome-based FZHY treatment show that FZHY has a better effect on LKYDS and LSS than on the other TCM syndromes, and the metabolic mechanisms might be involved in the increased detoxification function in LKYDS and the improvement of energy supply in LSS, which provides important evidence for the clinical application of TCM syndrome-based treatment.
\end{abstract}

Keywords: liver cirrhosis; Fuzheng-Huayu formula; traditional Chinese medicine syndrome-based treatment; metabolomics

Acta Pharmacologica Sinica (2018) 39: 942-951; doi: 10.1038/aps.2017.101; published online 26 Oct 2017

\section{Introduction}

Liver cirrhosis (LC) is defined as the histological development of regenerative nodules surrounded by fibrous bands resulting from chronic liver injury ${ }^{[1]}$. It is an advanced stage of liver fibrosis that is accompanied by the distortion of the hepatic vasculature. LC is the 14th most common cause of death worldwide, and it is regarded as an end-stage disease that invariably leads to clinical death unless liver transplantation is conducted ${ }^{[2]}$. Therefore, the treatment of LC is an urgent problem.

\footnotetext{
* To whom correspondence should be addressed.

E-mail shibingsu07@163.com (Shi-bing SU); liuliver@vip.sina.com (Ping LIU)

Received 2017-04-13 Accepted 2017-06-30
}

Chinese herbal formula (CHF), a herbal mixture prescription, is used for the treatment of special diseases and syndromes by regulating multiple pathways and targets. Fuzheng-Huayu formula (FZHY), a type of CHF, consists of six Chinese herbs, including Semen Persicae (Tao-ren), Cordyceps sinensis (Chong-cao), Gynostemma Pentaphyllammak (Jiaogulan), Radix Salvia Miltiorrhizae (Dan-shen), Pollen Pini (Songhuafen) and Fructus Schisandrae Chinensis (Wu-weizi), and has been proven effective in treating liver fibrosis and cirrhosis in both clinical trials ${ }^{[3-5]}$ and animal experiments ${ }^{[6-8]}$. It has been reported that FZHY could halt the development of liver fibrosis by inhibiting the activation of hepatic stellate cells (HSCs) ${ }^{[9]}$; however, the pharmacological mechanisms of FZHY on liver cirrhosis are still unclear. 
Traditional Chinese medicine (TCM) syndrome, also called ZHENG, is the basic concept of the TCM theory, which is a profile of symptoms and signs as a series of clinical phenotypes $^{[10]}$. TCM syndrome plays a vital role in understanding human homeostasis and guiding the applications of $\mathrm{CHF}^{[11]}$. Importantly, clinical investigations revealed that the total effective rate for LC was significantly higher in patients subject to integrated TCM syndrome-based treatment and Western medicine treatment than in patients only receiving Western medicine treatment ${ }^{[12]}$. We have reported that TCM syndrome-based FZHY treatment improved the life quality of LC patients ${ }^{[4]}$; however, the effective mechanisms of FZHY on LC are still uncertain.

Metabolomics is a kind of quantitative technology for measuring the multi-parametric, metabolic response of living systems $^{[13]}$. This technology has been widely used to study pathophysiological changes associated with various disease ${ }^{[14]}$. It can provide abundant information about the diagnosis of disease and the effects of drug treatment by evaluating a variety of endogenous substances that are differentially expressed in tissues or body fluid, such as the blood and urine ${ }^{[15]}$. In this study, we analyzed the metabolic profiles of FZHY in LC patients with TCM syndromes and different stages, aiming to explore the metabolic mechanisms of TCM syndrome-based FZHY efficacy in LC.

\section{Materials and methods Study population}

One hundred and thirteen participants, including 50 healthy controls and 63 LC patients, were recruited in the study at Shuguang, Yueyang, Longhua, and Putuo hospitals in Shanghai, China. FZHY (SFDA approval no: Z2005050546, 0.4 g per tablet) was provided by Shanghai Sundise Medicine Technology Development Co, Ltd (Shanghai, China). LC patients were given FZHY every day (1.6 g 3 times/day orally) for a total of 6 months. The protocol was approved by the Ethics Committees of the hospitals and conformed to the ethical guidelines of the Declaration of Helsinki (1964). An informed consent form was signed by all the participants.

\section{TCM syndromes and evaluation of their effects}

LC cases were classified into five TCM syndrome groups according to the diagnosis and differentiation of the TCM syndromes ${ }^{[16]}$, including liver stagnation syndrome (LSS, $n=17)$, spleen deficiency and damp overabundance syndrome (SDDOS, $n=10)$, damp-heat accumulation syndrome (DHAS, $n=11$ ), liver-kidney Yin deficiency syndrome (LKYDS, $n=14$ ), and blood stagnation syndrome (BSS, $n=11$ ).

Clinical symptoms and signs of LC patients were used to evaluate the effect of FZHY, including 45 clinical symptoms, such as no appetite, a tired and weak body, and a pale, white or dark and sluggish face, and 39 signs, such as a floating or heavy pulse and a dark or bruised tongue (Supplementary Table 1). Each clinical symptom and sign was a standard to quantify the degree of clinical manifestation of LC, which was called the TCM syndrome score. The TCM syndrome
Table 1. Programmed temperature of GC/MS.

\begin{tabular}{lcc}
\hline Rate $\left({ }^{\circ} \mathrm{C} / \mathrm{min}\right)$ & Temperature $\left({ }^{\circ} \mathrm{C}\right)$ & Hold time $(\mathrm{min})$ \\
\hline & 80 & 2 \\
5 & 93 & 0 \\
8 & 109 & 0 \\
5 & 185 & 2 \\
10 & 255 & 1 \\
10 & 290 & 7 \\
PostRun & 300 & 5 \\
\hline
\end{tabular}

score was defined as without, 0 ; weakness, 1 ; slightly heavy, 2 ; or very heavy, 3, and the signs were defined as within, 1; or without, 2 . To ensure the repeatability and reliability of the TCM syndrome and symptom diagnoses, all patients were diagnosed by 3 senior TCM physicians separately in the same condition, and the final diagnosis was made by a TCM chief physician. The final diagnosis was used for further analyses when the diagnoses were consistent.

\section{Sample preparation}

The fasting blood samples were collected from healthy controls and LC patients before breakfast and allowed to stand for $30 \mathrm{~min}$ at room temperature, and then they were centrifuged at $12000 \mathrm{r} / \mathrm{min}$ for $10 \mathrm{~min}$ at $4{ }^{\circ} \mathrm{C}$. All the serum samples were stored at $-80^{\circ} \mathrm{C}$ until further analysis was carried out.

\section{Laboratory investigations}

The serum levels of alanine aminotransferase (ALT), aspartate aminotransferase (AST), y-glutamyltransferase (GGT), albumin (ALB), total bilirubin (TBIL), triglyceride (TG), and hyaluronic acid (HA) were detected in the healthy control group, LC group and FZHY group with 6 months of treatment. The Child-Pugh scores were calculated according to clinical biochemical indices, the degree of hepatic encephalopathy, ascites, TBIL, ALB and prothrombin time.

\section{Metabolomics}

One hundred microliters of serum were transferred into a centrifuge tube. Next, an appropriate amount of methanol was added, and the mixture was vortex mixed for $1 \mathrm{~min}$. The serum was allowed to sit for $10 \mathrm{~min}$ at $-20{ }^{\circ} \mathrm{C}$ for protein precipitation, followed by centrifugation at $12000 \times \mathrm{g}$ for $10 \mathrm{~min}$ at $4{ }^{\circ} \mathrm{C}$, and then, $300 \mu \mathrm{L}$ of the supernatant was transferred to an autosampler vial and blown to dryness with $\mathrm{N}_{2}$. The residue was dissolved in $80 \mu \mathrm{L}$ of methoxyamine $(15 \mathrm{mg} / \mathrm{mL})$ for methoximation reaction, and $50 \mu \mathrm{L}$ of BSTFA containing $1 \%$ TMCS was added for thimethylsilylation. Finally, $1 \mu \mathrm{L}$ of the sample was injected into an Agilent 6890 gas chromatography (GC) system coupled with a 5975B mass spectrometer (MS) (Agilent Technologies, USA) for analysis.

The method of separation referred to our previous work ${ }^{[17]}$. Chromatographic separation was performed on a capillary column (Agilent J\&W DB-5ms Ultra Inert, $30 \mathrm{~m} \times 0.25 \mathrm{~mm} \times 0.25$ 
$\mu \mathrm{m})$ using an appropriate programmed temperature as listed in Table 1. The parameters set for mass spectrometry were as follows: scan range, $m / z \quad 30-550$ and temperatures of injection, interface and source, $280^{\circ} \mathrm{C}, 260^{\circ} \mathrm{C}$ and $230^{\circ} \mathrm{C}$, respectively.

\section{Data analysis}

SPSS 16.0 (Chicago, IL, USA) was used to carry out statistical analysis, and GraphPad Prism 5.0 (San Diego, California, USA) was used for statistical charts. Metabolite identification and quantification were carried out using the Agilent MSD workstation. Normalized data were imported into SIMCAP 11 (Umetrics AB, Umea, Sweden) for partial least squarediscriminant analysis (PLS-DA) and the calculation of variable importance in the projection (VIP). Pathway analysis was carried out using the KEGG database (http:/ / www.kegg.jp/).

\section{Results}

\section{Biochemical findings in LC treated by FZHY}

To evaluate the effect of FZHY on LC, the data from the biochemical tests were collected and analyzed (Figure 1).
Compared with the healthy group, most parameters were significantly higher in the LC group, except ALB $(P<0.05)$. Compared with the LC group, no significant differences were found in most parameters $(P>0.05)$, but ALT and AST were lower in the FZHY group. Notably, HA, a biochemical index related to liver fibrosis, was significantly lower in the FZHY group than in the LC group $(P<0.01)$, implying that FZHY could attenuate the degree of liver fibrosis in LC patients. Furthermore, the TCM syndrome scores were also significantly lower in the FZHY group than in the LC group $(P<0.01)$, suggesting that FZHY could also alleviate clinical symptoms related to TCM syndrome and improve quality of life (Supplementary Table S2).

\section{Effects of FZHY on TCM syndromes in LC}

Considering of the significant changes in the TCM syndrome scores, the effects of FZHY were further evaluated in LC patients with different TCM syndromes. As shown in Figure 2 and Supplementary Table S2, the TCM syndrome scores significantly decreased in the LSS group and the LKYDS
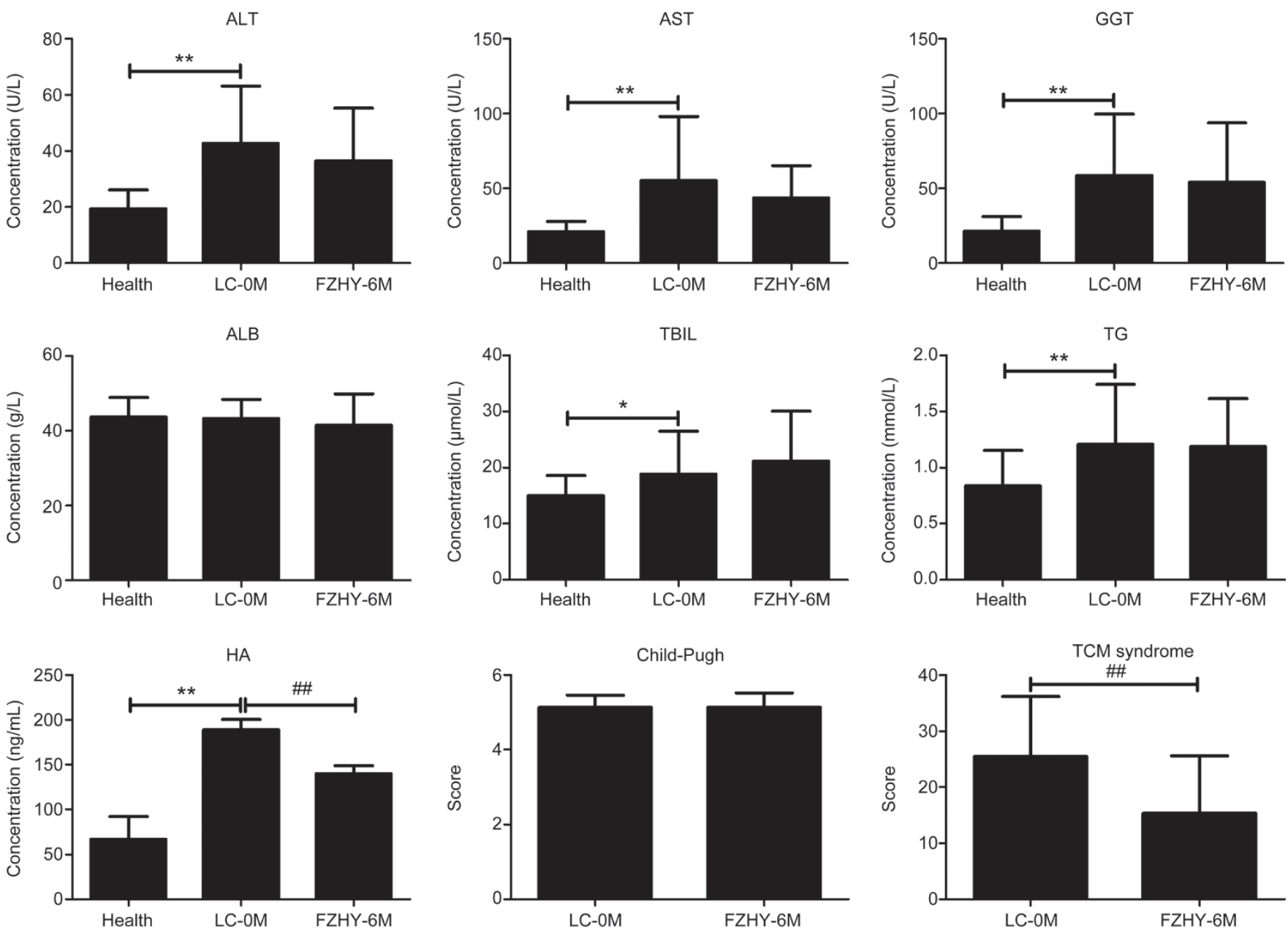

Figure 1. Clinical parameters of healthy controls, LC patients without and with six month-treatment of FZHY. $n=50$ in healthy group, and $n=63$ in LC group. Values were expressed as mean \pm SD. Significant differences were analyzed by One-Way ANOVA test or Student's $t$ test. Compared with healthy group, ${ }^{*} P<0.05,{ }^{* *} P<0.01$ compared with LC group. ${ }^{\# \#} P<0.01$. 


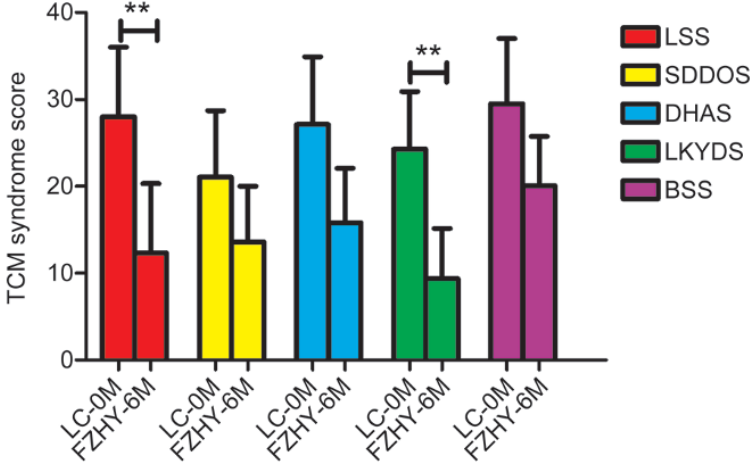

Figure 2. TCM syndrome scores of LSS group, SDDOS group, DHAS group, LKYDS group and BSS group before and after FZHY treatment. TCM syndrome scores were calculated according to 45 clinical symptoms and 39 signs. $n=17$ in LSS group, $n=10$ in SDDOS group, $n=11$ in DHAS group, $n=14$ in LKYDS group and $n=11$ in BSS group. Compared with LC group. ${ }^{* *} P<0.01$.

group after treatment with FZHY $(P<0.01)$, indicating that the effects of FZHY on LSS and LKYDS were better than on other
TCM syndromes.

Changes in the metabolic profiles of LC patients with TCM syndromes treated by FZHY

To explore the changes in the metabolic profiles of LC patients treated by different courses of FZHY, PLS-DA was performed using GC/MS data. Figure 3 shows that the healthy group was located on the left of the scatter plot, the LC group on the right, and the FZHY groups in the middle. With the extension of treatment time, the metabolic profiles of the FZHY groups moved from right to left, or closer to the healthy group and further away from the LC group. When the LC patients were treated for one month and three months, the metabolic profiles were hardly discriminated from the LC group (LC-0M), but when they were treated after six months, the metabolic profile was obviously different from LC-OM and similar to the healthy group. This indicated that FZHY regulated the metabolic profiles of LC patients from a pathologic state to a healthy state.

Because of the better effects of FZHY on LSS and LKYDS, the LC patients were classified into three groups: the LSS group, the LKYDS group and the other TCM syndromes group. The changes in the metabolic profiles in different TCM syndromes

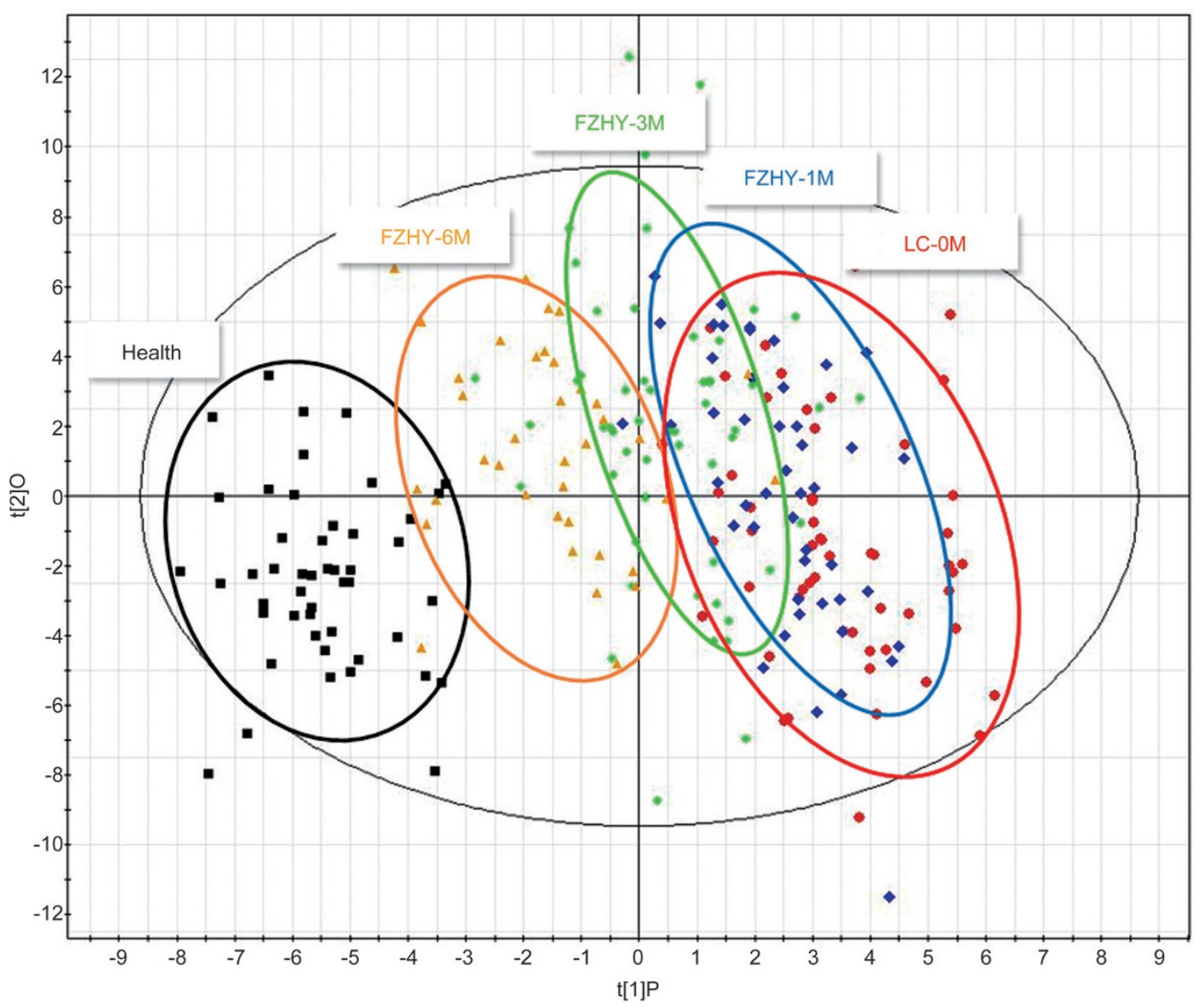

Figure 3. Dynamic changes of metabolomic profiling in healthy group, LC and FZHY groups with different time of FZHY treatment. PLS-DA analysis was performed and score plot was shown. Black referred to healthy group, red refers to LC group (LC-OM), blue referred to FZHY group with one month of FZHY treatment (LC-1M), green referred to FZHY group with three months of FZHY treatment (LC-3M), and orange referred to FZHY group with six months of FZHY treatment (LC-6M). 


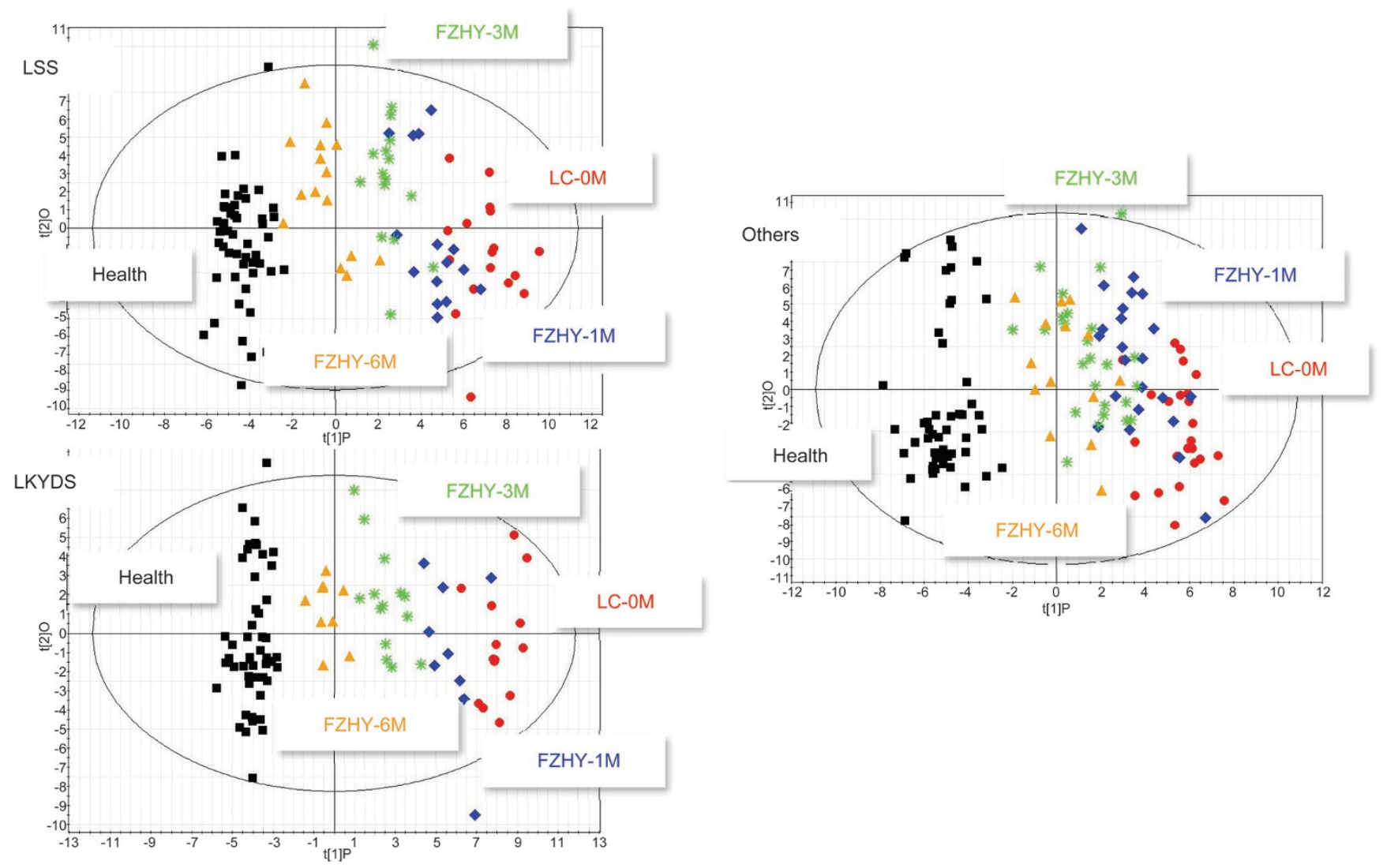

Figure 4. Dynamic changes of metabolomic profiling in LSS, LKYDS and the other TCM syndromes (others) by FZHY treatment. PLS-DA analysis was performed and score plots were shown. Black referred to healthy group, red referred to LC group (LC-OM), blue referred to FZHY group with one month of FZHY treatment (LC-1M), green referred to FZHY group with three months of FZHY treatment (LC-3M), and orange referred to FZHY group with six months of FZHY treatment (LC-6M).

groups were analyzed by the PLS-DA method. As shown in Figure 4, in the LSS and LKYDS groups, the metabolic profiles of the LC patients were restored from a pathological state to a healthy state following treatment with FZHY, while in the other TCM syndromes group, the metabolic profiles did not change significantly whether the LC patients were treated with FZHY or not.

Differently altered metabolites in the LC patients with TCM syndromes treated with FZHY

Metabolism in LC was regulated by FZHY treatment. The differently altered metabolites (DAMs) were defined as VIP >1 and $P<0.05$ (the VIP plot is shown in Supplementary Figure 1). Twenty-one DAMs were found between LC and the healthy groups or between FZHY and the LC groups, including carbohydrates, amino acids, fatty acids, etc (Supplementary Table 3). Among these, the dynamic changes of 18 metabolites are shown in Figure 5. Compared with the healthy controls, these metabolites significantly increased or decreased in LC patients before the treatment with FZHY (LC-0M); however, after the LC patients were treated with FZHY, most metabolites changed from a pathologic level to a healthy level. It is worth noting that most metabolites participating in amino acid metabolism, such as glycine, proline, tyrosine, glutamine, creatinine and urea, began to change when the LC patients took one month of FZHY, while most metabolites participating in carbohydrate metabolism, such as glucose, galactose, mannose, sorbitol, myoinositol and butanoic acid, and lipid metabolism, such as palmitic acid, stearic acid, linoleic acid, oleic acid, cholesterol and glutaric acid, began to change when LC patients took three or six months of FZHY, implying that amino acid metabolism might be regulated in early stages and that carbohydrate and lipid metabolism might be regulated in late stages.

Furthermore, DAMs were analyzed in different TCM syndromes. As shown in Figure 6, in the LSS group, there were 9 DAMs, including glucose, galactose, mannose, sorbitol, glycine, tyrosine, glutamine, stearic acid and linoleic acid. In the LKYDS group, there were also 9 DAMs, including sorbitol, myoinositol, butyric acid, glycine, proline, serine, glutamine, cystathionine and stearic acid. In the other TCM syndromes group, there were only 4 DAMs, including mannose, malonic acid, tyrosine and linoleic acid. The regulated metabolites in the other TCM syndromes group were much fewer than in the LSS group and LKYDS group, and this may be one of the reasons why the effect of FZHY on the other TCM syndromes 
Carbohydrate metabolism
Amino acid metabolism

Lipid metabolism
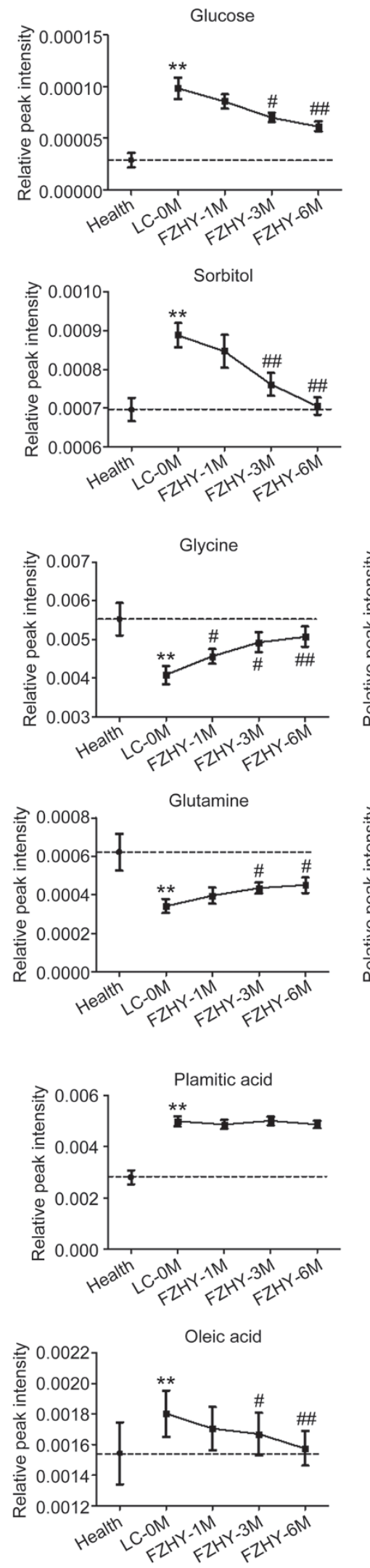
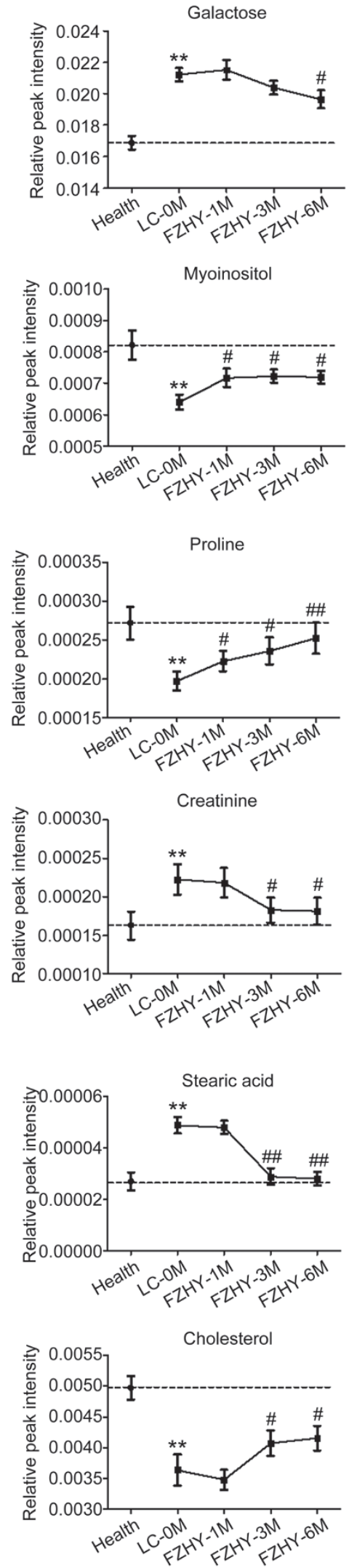
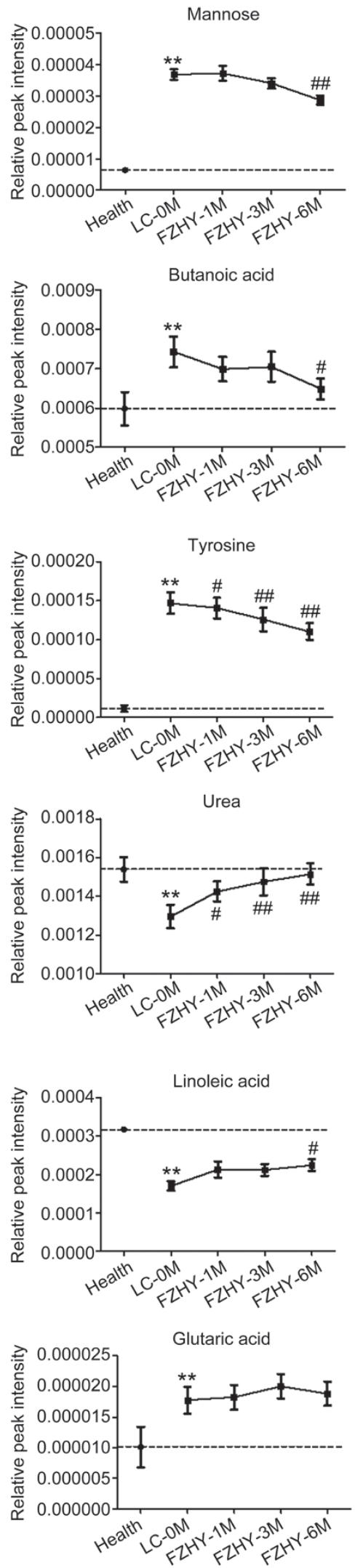

Figure 5. Dynamic changes of significantly altered metabolites among healthy group, LC group and FZHY groups, involved in carbohydrate, amino acid, and lipid metabolism. $n=50$ in healthy group and $n=63$ in LC group. Values were expressed as mean \pm standard deviation. Significant differences were analyzed by One-Way ANOVA test. Compared with healthy group, ${ }^{* *} P<0.01$; compared with LC group. ${ }^{\#} P<0.05,{ }^{\# \#} P<0.01$ vs FZHY-1M and FZHY-3M. 

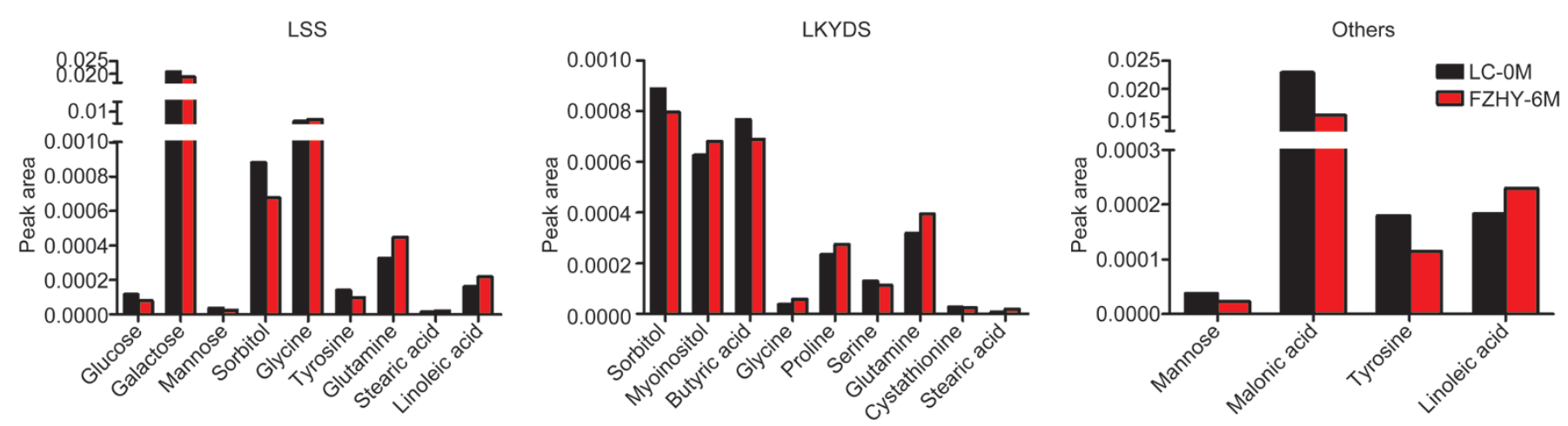

Figure 6. DAMs in different TCM syndromes. Nine DAMs were found in LSS group, nine DAMs were found in LKYDS group, and only four were found in the other TCM syndromes group.

was worse than on LSS and LKYDS.

\section{Altered metabolic pathways in LC patients with TCM syndromes treated with FZHY}

To explore the metabolic mechanism of FZHY, the metabolic pathways were analyzed through KEGG. Figure 7 shows the key pathways that included the altered metabolites. Red represents the metabolites changed when LC patients were treated with FZHY for one month, yellow represents the ones that changed in the third month, and green represents the ones that changed in the sixth month. Metabolites that changed in the first month mostly belonged to amino acid metabolism pathways and were involved in the conversion of amino acids and the body's detoxification processes, such as the urea cycle and the production of ammonia, which are located in the red box. Metabolites that changed in the third or sixth month mostly belonged to carbohydrate and lipid metabolism pathways and were involved in the body's energy supply processes, such as glycolysis and fatty acids metabolism, which are located in the green box. This suggests that the mecha-

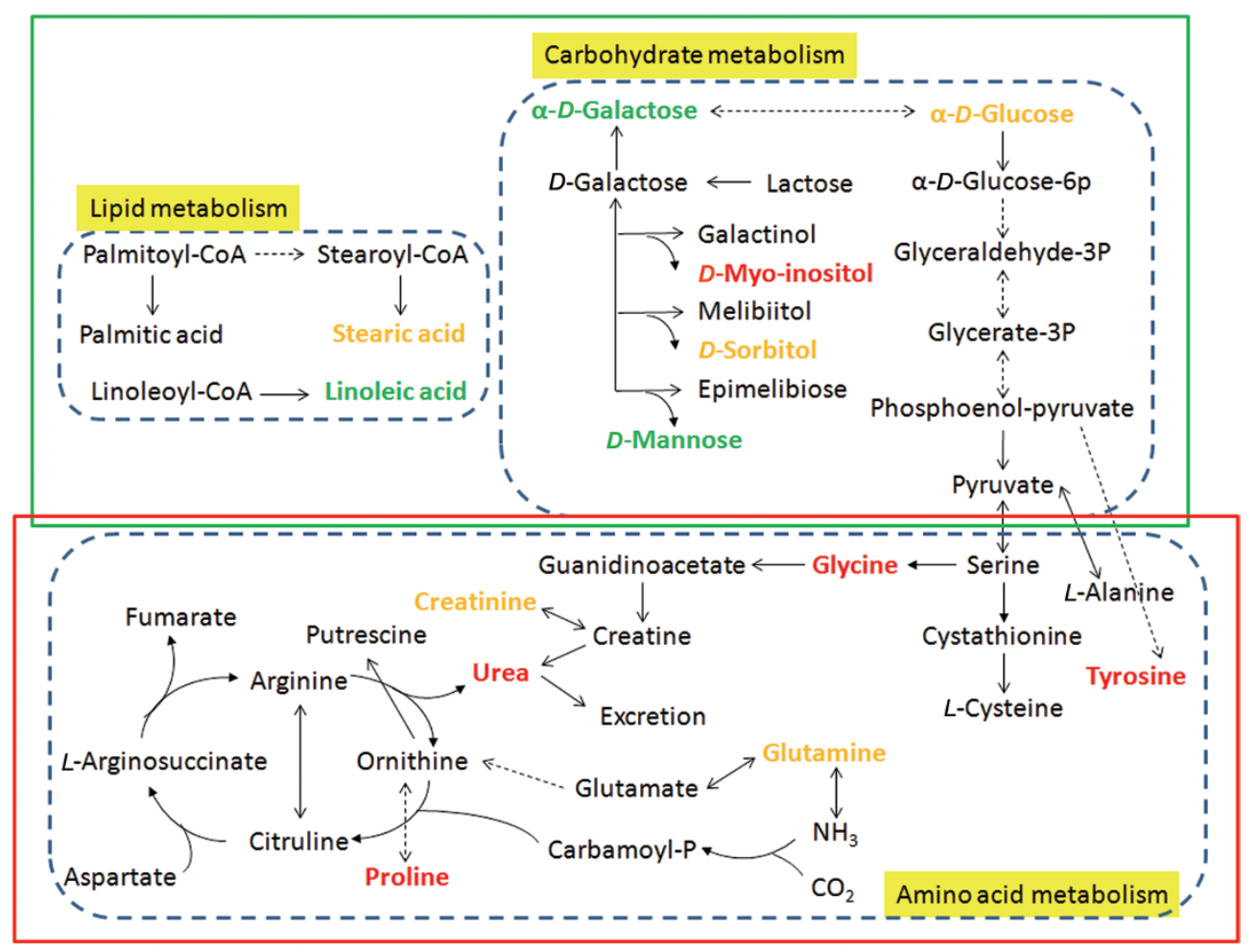

Figure 7. The network of significantly altered metabolic pathways in response to FZHY treatment. Red metabolites referred to ones significantly altered after LC patients took one month of FZHY, yellow metabolites referred to ones significantly altered after LC patients took three months of FZHY, and green metabolites refer to ones significantly altered after LC patients took six months of FZHY. Metabolites which changed in the first month mostly belonged to amino acid metabolism, located in red box. Metabolites which changed in the third or sixth month mostly belonged to carbohydrate and lipid metabolism, located in green box. 


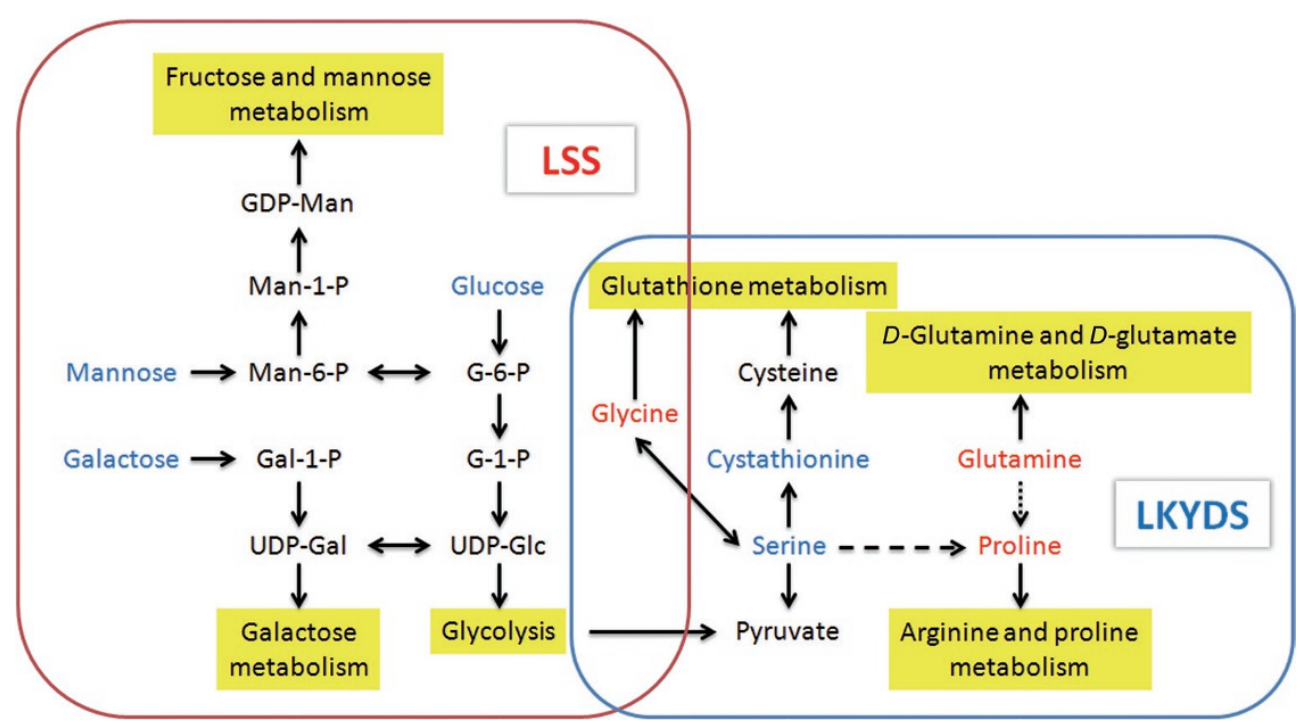

Figure 8. The network of significantly altered metabolic pathways in LSS and LKYDS. The significantly altered metabolic pathways in red box belong to LSS, which were mainly involved in carbohydrate metabolism and energy supply. The pathways in blue box belong to LKYDS, which were mainly involved in amino acid metabolism and detoxification. Red metabolites referred to ones significantly up-regulated by FZHY treatment, and blue metabolites referred to ones significantly down-regulated by FZHY treatment.

nism of FZHY mainly involves the solution of detoxification problems at first, followed by the improvement of the energy supply.

Considering the better effects of FZHY on the LSS and LKYDS, we further explored the network of significantly altered metabolic pathways in LSS and LKYDS. As shown in Figure 8, significantly altered metabolic pathways in LSS are located in the red box, including glycolysis, galactose metabolism, and fructose and mannose metabolism, which were mainly involved in carbohydrate metabolism and energy supply. Significantly altered metabolic pathways in LKYDS are located in the blue box, including glutathione metabolism, glutamine and glutamate metabolisms, and arginine and proline metabolisms, which were mainly involved in amino acid metabolism and detoxification. Red metabolites refer to ones that were significantly up-regulated by FZHY treatment, and blue metabolites refer to ones that were significantly downregulated by FZHY treatment. The results indicate that the metabolic mechanisms of FZHY on LSS may focus on the regulation of energy supply, and the mechanisms on LKYDS may focus on the solution of detoxification problems.

\section{Discussion}

As a Chinese herbal formula, FZHY has been reported to have effects in the prevention and treatment of liver fibrosis and cirrhosis through improved liver function, alleviated hepatic fibrosis, decreased Child-Pugh score and relieved TCM symptoms ${ }^{[18]}$. In this study, we found that with FZHY treatment, HA, a biochemical index related to liver fibrosis, was significantly lower than in LC patients $(P<0.01)$, and TCM syndrome scores also significantly decreased $(P<0.001)$, especially in LSS and LKYDS patients, indicating that the degree of fibrosis and clinical symptoms related to TCM syndrome were improved by FZHY treatment.

Previous studies have demonstrated that the mechanisms of FZHY against fibrosis might involve preventing HSCs from activation and proliferation, down-regulating the expression of collagen, protecting hepatocytes from apoptosis and facilitating their proliferation, etc $^{[0]}$; however, the mechanisms of FZHY against LC are still unclear. In the present study, we explored the mechanisms from the point-of-view of metabolic profiles and analyzed the pharmaceutical effects and metabolic mechanisms of TCM syndrome-based FZHY treatment in LC.

The liver has a detoxification function. Previous researchers have found that the hepatic detoxification capacity was lower and the urea synthesis rate and urea cycle enzyme activities were reduced in $\mathrm{LC}^{[19-21]}$. We found that FZHY could regulate the metabolic profiles of LC patients from a pathologic state to a healthy state, especially in LC patients with LSS and LKYDS. Interestingly, the metabolic pathways involved in the conversion of amino acids and the body's detoxification processes were regulated first. This implied that the detoxification problems of LC patients were urgent, and the mechanisms of FZHY against $\mathrm{LC}$ might be involved in the regulation of amino acids metabolism and the urea cycle. Notably, FZHY could regulate them and solve the detoxification problems in the initial stage of treatment.

LKYDS, a type of TCM syndrome, belongs to liver and kidney Yin deficiency syndrome, which is related to increased oxidative metabolism. When free radicals are increased by oxidative metabolism, the body and organs will be damaged. It has been reported that the content of lipid peroxide was upregulated in the blood of LKYDS patients, indicating that free radical reactions were enhanced in these patients ${ }^{[22]}$. In the present study, we found that FZHY could not only enhance detoxification functions but also regulated glutathione metab- 
olism in LKYDS, implying that FZHY-improved LKYDS in LC may involve the regulation of antioxidant pathways and the clearance of free radicals.

On the other hand, it has been shown that it is important to improve the decreased glycolysis in $\mathrm{LC}^{[23]}$. A previous study concluded that free fatty acids increased in parallel to increasing disease severity as defined by the Child-Pugh classification and could be a marker of energy malnutrition in $\mathrm{LC}^{[24]}$. This indicates that the energy supply of LC also needs to be improved, and the mechanisms of FZHY against LC might also involve the regulation of glycolysis and fatty acids metabolism.

LSS, a type of TCM syndrome, is the stagnation of Qi as the liver loses its ability to carry out catharsis, which is mostly related to emotional depression and low energy metabolism ${ }^{[25]}$. A previous study found that in LSS rats, most were sluggish, tired and inactive, and the intermediate products of the tricarboxylic acid cycle and glycolysis were decreased, such as citric acid and 2-oxpentanedioic acid, suggesting that the function of energy metabolism in the liver was weakened ${ }^{[26]}$. Considering that the mechanisms of FZHY against LC might involve the regulation of glycolysis and fatty acid metabolism, this might explain why energy metabolism pathways were significantly altered by FZHY treatment in LSS patients.

In conclusion, the metabolic mechanisms of FZHY-improved LC may refer to the regulation of the urea cycle, glycolysis and fatty acids metabolism; however, the solution of detoxification problems are addressed first. Remarkably, the evaluation of the effect of TCM syndrome-based FZHY treatment found that FZHY had a better effect on LKYDS and LSS than the other TCM syndromes, and the metabolic mechanisms might involve an increase in the detoxification function in LKYDS and an improvement in the energy supply in LSS, which provides some important evidence for the clinical application of TCM syndrome-based treatment.

\section{Acknowledgements}

This work was supported by the Key Program of the National Natural Science Foundation of China (No 81330084), the National Science and Technology Major Project of China (№ 2009ZX09311-003) and the E-institutes of Shanghai Municipal Education Commission (№ E 03008).

\section{Author contribution}

Shi-bing SU and Ping LIU desinged research; Ya-nan SONG and Fei-fei CAI performed research; Jian CHEN, Yi-yu LU, Qilong CHEN and Yong-yu ZHANG contributed new analytical tools and reagents; Ya-nan SONG analyzed data and wrote the paper.

\section{Supplementary information}

Supplementary information is available at the website of Acta Pharmacologica Sinica.

\section{References}

1 Schuppan D, Afdhal NH. Liver cirrhosis. Lancet 2008; 371: 838-51.
2 Tsochatzis EA, Bosch J, Burroughs AK. Liver cirrhosis. Lancet 2014; 383: $1749-61$.

3 Liu P, Liu C, Xu LM, Hu YY, Xue HM, et al. Effects of Fuzheng Huayu 319 recipe on liver fibrosis in chronic hepatitis $B$. World J Gastroenterol 1998; 4: 348-53.

4 Song YN, Sun JJ, Lu YY, Xu LM, Gao YQ, et al. Therapeutic efficacy of fuzheng-huayu tablet based traditional chinese medicine syndrome differentiation on hepatitis-B-caused cirrhosis: a multicenter doubleblind randomized controlled trail. Evid Based Complement Alternat Med 2013; 2013: 709305.

5 Chen Q, Wu F, Wang M, Dong S, Liu Y, Lu Y, et al. Transcriptional profiling and mirna-target network analysis identify potential biomarkers for efficacy evaluation of Fuzheng-Huayu formula-treated hepatitis B caused liver cirrhosis. Int J Mol Sci 2016; 17: 883.

6 Li XM, Peng JH, Sun ZL, Tian HJ, Duan XH, et al. Chinese medicine CGA formula ameliorates $\mathrm{DMN}$-induced liver fibrosis in rats via inhibiting MMP2/9, TIMP1/2 and the TGF-beta/Smad signaling pathways. Acta Pharmacol Sin 2016; 37: 783-93.

7 Yang $\mathrm{T}$, Liu S, Wang $\mathrm{CH}$, Tao YY, Zhou H, et al. Comparative pharmacokinetic and tissue distribution profiles of four major bioactive components in normal and hepatic fibrosis rats after oral administration of Fuzheng Huayu recipe. J Pharm Biomed Anal 2015; 114: 152-8.

8 Tao YY, Yan XC, Zhou T, Shen L, Liu ZL, Liu CH. Fuzheng Huayu recipe alleviates hepatic fibrosis via inhibiting TNF-alpha induced hepatocyte apoptosis. BMC Complement Altern Med 2014; 14: 449.

9 Pan Q, Wang YQ, Li GM, Duan XY, Fan JG. Fuzheng Huayu Recipe ameliorates liver fibrosis by restoring balance between epithelial-tomesenchymal transition and mesenchymal-to-epithelial transition in hepatic stellate cells. Biomed Res Int 2015; 2015: 935903.

10 Wang X, Xie G, Wang X, Zhou M, Yu H, Lin Y. Urinary metabolite profiling offers potential for differentiation of liver-kidney yin deficiency and dampness-heat internal smoldering syndromes in posthepatitis B cirrhosis patients. Evid Based Complement Alternat Med 2015; 2015: 464969 .

11 Song YN, Zhang H, Guan Y, Peng JH, Lu YY, Hu YY, et al. Classification of traditional Chinese medicine syndromes in patients with chronic hepatitis $b$ by seldi-based proteinchip analysis. Evid Based Complement Alternat Med 2012; 2012: 626320.

12 Zhou HP. Clinical study of liver cirrhosis treated by integrated traditional Chinese and western medicine. Chin J Integr Tradit Western Med 2013; 21: 199-201.

13 Nicholson JK, Lindon JC, Holmes E. 'Metabonomics': understanding the metabolic responses of living systems to pathophysiological stimuli via multivariate statistical analysis of biological NMR spectroscopic data. Xenobiotica 1999; 29: 1181-9.

14 Goodacre R. Metabolomics of a superorganism. J Nutr 137: 259S-266S.

15 Chen T, Xie G, Wang X, Fan J, Qiu Y, Zheng X, et al. Serum and urine metabolite profiling reveals potential biomarkers of human hepatocellular carcinoma. Mol Cell Proteomics 2007; 10: M110 004945.

16 Chinese Association of Integrative Medicine LDC. Guideline for the diagnosis and treatment of liver fibrosis with integrative medicine. J Chin Integr Med 2006; 4: 551-5.

17 Song YN, Dong S, Wei B, Liu P, Zhang YY, Su SB. Metabolomic mechanisms of gypenoside against liver fibrosis in rats: An integrative analysis of proteomics and metabolomics data. PLoS One 2017; 12: e0173598.

18 Dong S, Chen QL, Su SB. Curative effects of Fuzheng Huayu on liver fibrosis and cirrhosis: a meta-analysis. Evid Based Complement 
Alternat Med 2015; 2015: 125659.

19 Muting D, Kalk JF, Fischer R, Wuzel H, Reikowski J. Hepatic detoxification and hepatic function in chronic active hepatitis with and without cirrhosis. Dig Dis Sci 1988; 33: 41-6.

20 Ukida M, Takao T. Amino acid metabolism in liver cirrhosis. Nihon Rinsho 1992; 50: 1604-8.

21 Maier KP, Talke H, Gerok W. Activities of urea-cycle enzymes in chronic liver disease. Klin Wochenschr 1979; 57: 661-5.

22 Ren XQ, Jiang SQ, Lu YQ, Luo XP, Guo HQ, et al. The relationship of liver kidney Yin deficiency syndrome, blood lipids, energy metabolism and free radical reaction. Henan Tradit Chin Med 1996; 16: 346-8.

23 Kasahara H, Ohyanagi H, Saitoh Y. Changes of gluconeogenesis and alanine metabolism following partial hepatectomy in normal and cirrhotic rats. Nihon Geka Gakkai Zasshi 1988; 89: 365-75.

24 Hanai T, Shiraki M, Nishimura K, Imai K, Suetsugu A, et al. Free fatty acid as a marker of energy malnutrition in liver cirrhosis. Hepatol Res 2014; 44: 218-28.

25 Xiao M, Zhou YY. Comparison of behavior indexes of different kinds of liver-qi stagnation anamal models. Chin J Tradit Med Sci Technol 2015; 22: 123-4.

26 Wang WM, Huang YH, Xiong ZF, Xu ZX, Sheng GG. Study on urine metabonomics for rat model with stagnation of liver-Qi syndrome. Chin J Integr Tradit Western Med Liver Dis 2010; 20: 102-5. 